Revisão / Review

\title{
Deficiência de ferro na doença renal crônica
}

Iron deficiency in chronic kidney disease

Hugo Abensur

\begin{abstract}
Anemia é uma complicação quase universal nos pacientes em estágios avançados de doença renal crônica (DRC). Ela está associada com maior número de internações hospitalares, maior mortalidade e pior qualidade de vida dos pacientes. Ela tem várias causas, sendo deficiência de eritropoetina e ferro as duas principais causas. A condição inflamatória presente na DRC interfere com a ação da eritropoetina e com a absorção intestinal de ferro e mobilização de ferro dos estoques, devido ao aumento de hepcidina. A correção parcial (não completa) da anemia promove melhores resultados nos pacientes com DRC. Rev. Bras. Hematol. Hemoter. 2010; 32(Supl.2):84-88.
\end{abstract}

Palavras-chave: Anemia; eritropoetina; deficiência de ferro; doença crônica; diálise.

\section{Introdução}

A doença renal crônica (DRC) é caracterizada por perda lenta e progressiva da função renal. Segundo a Diretriz Brasileira de DRC, ${ }^{1}$ ela é classificada em cinco estágios de acordo com o grau de perda da função renal, conforme exposto na Tabela 1 .

A anemia surge precocemente no curso da DRC e sua prevalência aumenta à medida que ocorre diminuição da função renal.

Em estudo multicêntrico brasileiro, ${ }^{2}$ executado em ambulatórios de tratamento conservador de DRC, considerando anemia como concentração de hemoglobina menor que $11 \mathrm{~g} / \mathrm{dL}$, a prevalência de anemia foi de $8 \%, 13 \%, 20 \% \mathrm{e}$ $39 \%$, respectivamente nos estágios de DRC de 2 a 5 . Porém, como alguns destes pacientes já estavam sendo tratados com reposição de ferro e eritropoetina, a prevalência de anemia ainda seria maior.

No estudo multicêntrico canadense envolvendo 446 pacientes com DRC sem uso de eritropoetina, a prevalência de anemia no estágio 5 de DRC foi em torno de $90 \%$ quando considerado anêmicos pacientes com hemoglobina menor que $13 \mathrm{~g} / \mathrm{dL} .^{3}$
Tabela 1. Classificação da DRC ${ }^{1}$

\begin{tabular}{ccc}
\hline Estágio & $\begin{array}{c}\text { Filtração glomerular } \\
(\mathrm{mL} / \mathrm{min})\end{array}$ & Grau de insuficiência renal \\
\hline 0 & $>90$ & $\begin{array}{c}\text { Grupos de risco para DRC } \\
\text { Ausência de lesão renal } \\
\text { Lesão renal com } \\
\text { função renal normal } \\
1\end{array}$ \\
2 & $60-89$ & IR leve ou funcional \\
3 & $30-59$ & IR moderada ou laboratorial \\
4 & $15-29$ & IR severa ou clínica \\
5 & $<15$ & IR terminal ou dialítica \\
\hline
\end{tabular}

$\mathrm{DRC}=$ doença renal crônica; IR = insuficiência renal

O estudo NHANES III, ${ }^{4}$ que envolveu cerca de quase 20 milhões de pacientes com algum grau de DRC, considerando anemia como hemoglobina menor que $13 \mathrm{~g} / \mathrm{dL}$, mostrou que a incidência de anemia é menor que $10 \%$ nos estágios 1 e 2 de DRC, $20 \%-40 \%$ no estágio $3,50 \%-60 \%$ no estágio 4 , e mais de $70 \%$ no estágio 5 de DRC.

A prevalência de deficiência de ferro é muito comum na DRC e é em torno de $50 \%,{ }^{5}$ sendo, portanto, ao lado da defi-

Nefrologista. Médico Assistente de Nefrologia do Hospital das Clínicas da Faculdade de Medicina da Universidade de São Paulo - HC FMUSP.

Hospital das Clinicas da Faculdade de Medicina da Universidade de São Paulo - São Paulo-SP.

Correspondência: Hugo Abensur

AV. Масисо 58 cj 11 - Moema

04523000 - São Paulo, SP - Brasil

Tel/Fax.: (55 11) 3283-5304

E-mail:sabensur@usp.br

Doi: 10.1590/S1516-84842010005000047 
ciência relativa de eritropoetina, as duas principais causas de anemia na DRC.

\section{Fisiopatologia da anemia na DRC}

A anemia na DRC é caracteristicamente normocrômica, normocítica e com contagem de células vermelhas na medula óssea normal ou diminuída, devido ao seu caráter hipoproliferativo. Sua principal causa é a deficiência de eritropoetina, devido à perda de massa renal, sítio principal de produção de eritropoetina.

A deficiência de eritropoetina é relativa na DRC. Num clássico experimento feito em ovelhas foi demonstrado que os níveis séricos de eritropoetina de ovelhas portadoras de DRC são semelhantes aos de ovelhas com função renal normal, porém diminuídos frente ao grau de anemia que elas apresentavam. Uma ovelha com função renal normal e anemia apresenta níveis bem mais elevados de eritropoetina circulante. ${ }^{6}$

A etiologia da anemia na DRC é multifatorial, sendo que diversos fatores concorrem para o advento da anemia na DRC, tais como: deficiência absoluta ou funcional de ferro, que está presente em 30\% a 50\% dos pacientes com DRC; 5 perda sanguínea; hiperparatireoidismo; estado inflamatório; diminuição da meia-vida das hemácias; e deficiência de ácido fólico e/ou vitamina B12.

A doença renal crônica é um estado inflamatório. Os níveis de proteína $\mathrm{C}$ reativa aumentam à medida que os pacientes perdem função renal. ${ }^{7}$ Este estado inflamatório está associado com a presença de anemia. Citocinas pró-inflamatórias, como a interleucina ${ }^{6}$ e o fator de necrose tumoral, atuam nas células progenitoras eritropoéticas, de maneira oposta à eritropoeitina, estimulando a apoptose. ${ }^{8} \mathrm{O}$ estado inflamatório da DRC provoca uma situação de resistência à ação medular da eritropoetina. Quando pacientes portadores de DRC apresentam infecções concomitantes, existe um claro agravamento da anemia.

Na situação de inflamação ocorre aumento da produção hepática de hepcidina, um peptídeo que inibe a absorção duodenal de ferro e a mobilização de ferro dos seus estoques (células do sistema reticuloendotelial - SRE). Portanto, na DRC é muito comum a ocorrência de deficiência de ferro absoluta ou funcional. A deficiência absoluta de ferro pode ser causada por diminuição da absorção intestinal de ferro (hepcidina, desnutrição) ou por perdas sanguíneas. É caracterizada por níveis séricos baixos de ferro, saturação diminuída de transferrina e níveis baixos de ferritina. A deficiência funcional de ferro é devida à menor mobilização de ferro dos macrófagos do SRE quando os níveis de hepcidina estão aumentados. Ela é caracterizada por níveis elevados de ferritina e saturação de transferrina diminuída. Pelo exposto acima é fácil entender a importância da reposição de ferro, além da eritropoetina, no tratamento da anemia relacionada à DRC. ${ }^{9}$
Os pacientes portadores de DRC apresentam perdas sanguíneas com maior frequência devido à agregação plaquetária deficiente por alteração do fator VIII de von Villebrand. Esta perda pode ser intestinal, pois os pacientes com DRC apresentam não raramente displasias vasculares na via digestiva. Além disto, os pacientes em programa de hemodiálise recebem heparina para evitar coagulação do sistema extracorpóreo e perdem sangue nas linhas e filtros de hemodiálise. A perda sanguínea crônica nestes pacientes concorre para o advento de anemia ferropriva.

O hiperparatireoisdimo secundário é uma complicação comum na DRC, que também contribui para o advento de anemia. Na osteíte fibrosa cística, causada pelo hiperparatireoidismo secundário, ocorre substituição de parte da medula óssea por fibrose, com diminuição de massa medular. Na osteíte fibrosa cística existe aumento da expressão medular de diversas citocinas, que também pode contribuir para uma maior resistência à ação da eritropoetina. ${ }^{10}$

Vários estudos evidenciaram menor sobrevida dos eritrócitos na DRC. ${ }^{11}$ A etiologia não é bem definida, alguns autores sugerem um certo grau de hiperesplenismo na DRC.

Os pacientes portadores de $\mathrm{DRC}$, devido às restrições alimentares a que são submetidos, à perda de apetite inerente à condição patológica e perdas durante o processo de diálise, apresentam, com alguma frequência, deficiência de vitaminas do complexo B e ácido fólico, que pode contribuir para o advento de anemia.

$\mathrm{O}$ uso de inibidores do sistema renina angiotensina, provocando diminuição da concentração de angiotensina II ou inibição da ação da angiotensina II, pode contribuir para anemia da DRC, pois a angiotensina II tem efeito estimulador da eritropoese na medula óssea. ${ }^{12}$

\section{Repercussões clínicas}

A anemia é uma grave consequência da DRC. Provoca incapacidade física e mental, sendo responsável pela redução da sobrevida e da qualidade de vida ${ }^{13}$ dos pacientes portadores desta condição clínica; além disso, ela estigmatiza o paciente portador de DRC, pois acarreta palidez cutânea, conferindo-lhe um aspecto de doente, prejudicando de maneira importante a sua recuperação social.

As doenças cardiovasculares são as principais causas de mortalidade na DRC. A anemia contribui decisivamente para o agravamento da hipertrofia ventricular esquerda (HVE) e da insuficiência cardíaca congestiva (ICC). A anemia também contribui para o agravamento dos sintomas de angina de pacientes portadores de DRC e doenças coronarianas.

Nos pacientes com DRC, a queda de $0,5 \mathrm{~g} / \mathrm{dL}$ de hemoglobina está associada com um aumento de $32 \%$ no risco de desenvolvimento de HVE. ${ }^{14}$ Um estudo prospectivo envolvendo 433 pacientes em programa de hemodiálise mostrou que a queda de $1 \mathrm{~g} / \mathrm{dL}$ de hemoglobina esteve associada a 
um aumento de 50\% no risco de ICC. ${ }^{15}$ A anemia também aumenta o risco de acidente vascular cerebral nos pacientes com DRC. ${ }^{16}$ Deste modo, níveis de hemoglobina menores que $11 \mathrm{~g} / \mathrm{dL}$ nos pacientes portadores de DRC estão associados com maior número de internações hospitalares e maior mortalidade. ${ }^{17}$

O estudo RENAAL mostrou associação entre anemia e progressão da DRC. ${ }^{18}$ Pacientes com DRC anêmicos têm perda mais acelerada da função renal e necessitam mais precocemente iniciar programa de diálise.

A anemia pode levar à necessidade de transfusões sanguíneas nos pacientes portadores de DRC. As transfusões sanguíneas, além do risco de infecções, podem provocar sensibilizações contra o sistema HLA e inviabilizar a realização de um futuro transplante renal, a melhor alternativa de substituição da função renal.

\section{Abordagem diagnóstica}

A anemia da DRC aparece a partir do estágio 3 de DRC. Portanto, pacientes anêmicos nos estágios 1 e 2 de DRC geralmente apresentam outras causas de anemia. Existe uma certa proporcionalidade entre o grau de anemia e o estágio

Tabela 2. Outras causas de anemia na $\mathrm{DR}^{19}$

Deficiência absoluta ou funcional de ferro
Subdiálise - diálise inadequada
Infecção
Microinflamação
Sangramento crônico
Osteíte fibrosa - hiperparatireoidismo
Hemoglobinopatias
Intoxicação por alumínio
Mieloma múltiplo
Neoplasias em geral
Deficiência de vitamina B12
Deficiência de folato
Desnutrição
Hemólise
Perda de sangue no circuito extracorpóreo
Efeitos adversos de alguns medicamentos
Presença de aloenxerto renal não funcionante

de DRC. Um paciente muito anêmico em estágio 3 de DRC certamente deve ter outra causa de anemia não associada à DRC. Para investigação da anemia é necessário um eritrograma completo, que pode evidenciar pistas de anemia ferropriva (presença de microcitose), de deficiência de vitamina B12 ou ácido fólico (presença de macrositose), de hemólise (presença de esquizócitos). A avaliação dos estoques de ferro é obrigatória, incluindo a dosagem da saturação de transferrina e de ferritina sérica. A saturação de transferrina sérica abaixo de $20 \%$ é considerada como deficiência de ferro. Os níveis de ferritina menores que $100 \mathrm{ng} / \mathrm{mL}$ são considerados como deficiência de ferro nos pacientes em tratamento conservador (estágio 3 e 4 de DRC) e em programa de diálise peritoneal. Em hemodiálise, níveis de ferritina menores que $200 \mathrm{ng} / \mathrm{mL}$ estão associados à deficiência de ferro. A Tabela 2 mostra outras causas de anemia na DRC. A abordagem diagnóstica da anemia na DRC pode ser encontrada na Diretriz para o Tratamento da Anemia no Paciente com Doença Renal Crônica. ${ }^{19}$

\section{Prevenção e considerações terapêuticas}

A prevenção da anemia na DRC consiste na própria prevenção da DRC. As duas principais causas de DRC são diabetes e hipertensão arterial. Estas duas situações estão associadas com hábitos alimentares inadequados, falta de atividade física e obesidade. Portanto, temos que prevenir o advento de diabetes e hipertensão arterial e tratar adequadamente os pacientes com diagnóstico de diabéticos e/ou de hipertensão arterial para prevenir o advento de DRC e a anemia associada.

Caso seja constatada deficiência de ferro, a reposição deve ser feita prontamente por via endovenosa nos pacientes em programa de hemodiálise, pois estes pacientes têm perda crônica de sangue e dificuldade de absorver ferro devido aos níveis aumentados de hepcidina, um peptídeo produzido no fígado por indução de citocinas inflamatórias, que bloqueia a absorção intestinal de ferro e a mobilização de ferro dos estoques. ${ }^{20}$

Nos pacientes em tratamento conservador e em programa de diálise peritoneal, por apresentarem menor perda de sangue, pode ser tentada inicialmente a via oral de administração de ferro; porém, a reposição endovenosa de ferro deverá ser iniciada em caso de não resposta à terapia oral. A reposição de ferro deverá ser interrompida quando a saturação de transferrina for superior a $40 \%$ e a concentração de ferritina sérica for superior à $500 \mathrm{ng} / \mathrm{mL} .{ }^{21} \mathrm{Na}$ Tabela 3 está exposto o esquema de reposição de ferro para pacientes em programa de hemodiálise de acordo com a Diretriz Brasileira para o Tratamento da Anemia no Paciente com Doença Renal Crônica. ${ }^{21}$ 
Uma vez repostos os estoques de ferro e os pacientes permanecendo com níveis de hemoglobina menores que $11 \mathrm{~g} / \mathrm{dL}$, deve-se iniciar o tratamento com medicamentos estimuladores de eritropoese, almejando níveis de hemoglobina entre $11 \mathrm{e} 12 \mathrm{~g} / \mathrm{dL}$, pois três grandes estudos prospectivos evidenciaram que níveis maiores de hemoglobina não trouxeram benefícios para os pacientes portadores de DRC e, inclusive, aumentaram os riscos de eventos cardiovasculares. $^{22-24}$

No Brasil dispomos de eritropoetinas convencionais com meia vida curta e necessidade de uma a três administrações semanais, preferencialmente subcutâneas, e, mais recentemente, passamos a dispor de CERA (betaepoetina metoxipolietilenoglicol) com meia vida mais longa, podendo ser administrada a cada 15 dias na fase de correção da hemoglobina e mensalmente na fase de manutenção por via subcutânea ou endovenosa. Os principais efeitos colaterais dos medicamentos estimuladores da eritropoese são o advento ou piora da hipertensão arterial e fenômenos trombóticos. Raramente pode ocorrer formação de anticorpos contra eritropoetina, levando ao quadro de aplasia pura de células vermelhas (APCV). AAPCV é um quadro muito grave, com necessidade de transfusões sanguíneas semanais, e deve ser tratada com a interrupção do tratamento com eritropoetina, adição de corticoterapia e imunossupressores.

Nos pacientes que não respondem com incremento satisfatório dos níveis de hemoglobina, deve-se procurar e sanar as causas de resistência; dentre elas ressaltam-se a deficiência de ferro, perdas sanguíneas, estados inflamatórios, infecciosos, hiperparatireoidismo, subdiálise, carências de vitaminas e hemólise. A deficiência funcional de ferro é uma situação associada à inflamação (aumento de hepcidina), em que o paciente apresenta estoque adequado de ferro no organismo, caracterizado por níveis elevados de ferritina sérica, mas não consegue mobilizar o ferro dos estoques, e a saturação de transferrina fica abaixo de $25 \%$. Nestes casos, o emprego de ferro endovenoso poderá contribuir para a melhora do quadro de resistência aos medicamentos estimulantes de eritropoetina, conforme demonstrado no estudo DRIVE. ${ }^{25}$

Com relação à terapia adjuvante, a reposição de vitamina B12 e ácido fólico é recomendada apenas em caso de deficiência destas substâncias. A suplementação de vitamina $C$, vitamina $E$, vitamina B6, carnitina, andrógeno, estatina e pentoxifilina não necessita ser feita rotineiramente como tratamento adjuvante da anemia de pacientes com DRC. ${ }^{26}$

\section{Conclusões}

A anemia é uma complicação da DRC, causada principalmente pela deficiência de eritropoetina. Porém, a deficiência de ferro é muito frequente na DRC. A reposição de ferro na DRC é imperiosa, pois, a reposição isolada de eritropoetina não funciona na situação de deficiência de ferro.

\section{Abstract}

Anemia is an almost universal complication of patients in advanced stages of chronic kidney disease (CKD). It is associated with more hospitalizations, increased mortality and worse quality of life. Although there are several causes, erythropoietin and iron deficiency are the most common. The inflammatory condition present in $C K D$ interferes with the action of erythropoietin, intestinal iron absorption and iron mobilization from deposits, due to increased hepcidin concentrations. Partial but incomplete correction of anemia promotes better outcomes in patients with CKD. Rev. Bras. Hematol. Hemoter. 2010;32(Supl.2):84-88.

Key words: Anemia; erythropoietin; iron deficiency; chronic disease; dialysis.

\section{Referências Bibliográficas}

1. Romão Jr JE. Doença renal crônica: Definição, Epidemiologia e Classificação. J Bras Nefrol. 2004;3(1):1-3.

2. Canziani MEF, Bastos MG, Bregman R, Pecoits Filho R, Tomiyama $\mathrm{C}$, Draibe SA, et al. Deficiência de ferro e anemia na doença renal crônica. J Bras Nefrol. 2006;28(2):86-90.

3. Middleton RJ, Parfrey PS, Foley RN. Left ventricular hypertrophy in the renal patient. J Am Soc Nephrol. 2001;12(5):1079-84.

4. Astor BC, Muntner P, Levin A, Eustace JA, Coresh J. Association of kidney function with anemia: the Third National Health and Nutrition Examination Survey (1988-1994). Arch Intern Med. 2002;162(12):1401-8.

5. Hutchinson FN, Jones WJ. A cost-effectiveness analysis of anemia screening before erythropoietin in patients with end-stage renal disease. Am J Kidney Dis. 1997;29(5):651-7.

6. Eschbach JW, Adamson JW, Dennis MB. Physiologic studies in normal and uremic sheep: I. The experimental model. Kidney Int. 1980;18(6):725-31.

7. Romão JE Jr, Haiashi AR, Elias RM, Luders C, Ferraboli R, Castro MC, et al. Positive acute-phase inflammatory markers in different stages of chronic kidney disease. Am J Nephrol. 2006;26(1):59-66.

8. De Maria R, Zeuner A, Eramo A, Domenichelli C, Bonci D, Grignani $\mathrm{F}$, et al. Negative regulation of erythropoiesis by caspasemediated cleavage of GATA-1. Nature. 1999;401(6752):489-93.

9. Weiss G, Goodnough LT. Anemia of chronic disease. N Engl J Med. 2005;352(10):1011-23.

10. Santos FR, Moysés RM, Montenegro FL, Jorgetti V, Noronha IL. IL-1beta, TNF-alpha, TGF-beta, and bFGF expression in bone biopsies before and after parathyroidectomy. Kidney Int. 2003; 63(3):899-907.

11. Rath RN, Das RK 4th, Panda RK, Mahakur AC, Patnaik SR. Red cell survival time in chronic renal failure. J Assoc Physicians India. 1979;27(10):969-74.

12. Mrug M, Stopka T, Julian BA, Prchal JF, Prchal JT. Angiotensin II stimulates proliferation of normal early erythroid progenitors. J Clin Invest. 1997;100(9):2310-4.

13. Valderrábano F. Erythropoietin in chronic renal failure. Kidney Int. 1996;50(4):1373-91.

14. Levin A, Thompson C, Ethier J, Carlisle E, Yobe S, Mendelssohn D, et al. Left ventricular mass index increase in early enal failure: impact of decline in hemoglobin. Am J Kidney Dis. 1999;34(1):125-34. 
15. Harnett JD, Kent GM, Foley RN, Parfrey OS. Cardiac function and hematocrit level. Am J Kidney Dis. 1995;25(4 Suppl 1):S3-7.

16. Abramson JL, Jurkovitz CT, Vaccarino V, Weintraub WS, McClellan W. Chronic kidney disease, anemia, and incident stroke in a middleaged, community-based population: the ARIC Study. Kidney Int. 2003;64(2):610-5.

17. Rayner HC, Pisoni RL, Bommer J, Canaud B, Hecking E, Locatelli $\mathrm{F}$, et al. Mortality and hospitalization in haemodialysis patients in five European countries: results from the Dialysis Outcomes and Practice Patterns Study (DOPPS). Nephrol Dial Transplant. 2004; 19(1):108-20.

18. Keane WF, Lyle PA. Reduction of Endpoints in NIDDM with the Angiotensin II Receptor Antagonist Losartan study. Recent advances in management of type 2 diabetes and nephropathy: lessons from the RENAAL study. Am J Kidney Dis. 2003;41(3 Suppl 1):S22-5.

19. Ribeiro-Alves MA, Gordan PA. Diagnóstico de anemia em pacientes portadores de doença renal crônica. J Bras Nefrol. 2007;29(4Sup1.4):4-6.

20. Fudin R, Jaichenko J, Shostak A, Bennett M, Gotloib L. Correction of uremic iron deficiency anemia in hemodialyzed patients: a prospective study. Nephron. 1998;79(3):299-305.

21. Abensur H, Castro MCM. Reposição de ferro no tratamento da anemia. J Bras Nefrol. 2007;29(4-Supl.4):9-11.

22. Drüeke TB, Locatelli F, Clyne N, Eckardt KU, Macdougall IC, Tsakiris D, et al; CREATE Investigators. Normalization of hemoglobin level in patients with chronic kidney disease and anemia. N Engl J Med. 2006;355(20):2071-84.

23. Singh AK, Szczech L, Tang KL, Barnhart H, Sapp S, Wolfson M, et al; CHOIR Investigators. Correction of anemia with epoetin alfa in chronic kidney disease. N Engl J Med. 2006; 355(20):2085-98.

24. Pfeffer MA, Burdmann EA, Chen CY, Coopre ME, de Zeeuw D, Eckardt KU, et al; TREAT Investigators. A trial of darbepoetin alfa type 2 diabetes and chronic kidney disease. N Engl J Med. 2009;361(21):2019-32.

25. Coyne DW, Kapoian T, Suki W, Singh AK, Moran JE, Dahl NV, et al; DRIVE Study Group: Ferric gluconate is highly efficacious in anemic hemodialysis patients with high serum ferritin an low transferrin saturation: results of the dialysis patients' response to IV iron with elevated ferritin (DRIVE) study. J Am Soc Nephrol. 2007;18(3):975-84.

Suporte financeiro: Este artigo faz parte de um suplemento de anemia ferropriva que teve o apoio financeiro da Nycomed.

O tema foi sugerido e avaliado pelo coeditor deste fascículo educativo, Rodolfo Delfini Cançado, e pelo board interno da RBHH, e publicado após a concordância do editor, Milton Artur Ruiz.

Recebido: $13 / 12 / 2009$

Aceito: $15 / 01 / 2010$ 